CERN-TH/97-28

hep-ph/9702431

\title{
WHAT IS THE MASS OF THE LIGHTEST SUPERSYMMETRIC HIGGS BOSON? ${ }^{a}$
}

\author{
PIOTR H. CHANKOWSKI ${ }^{b}$ \\ Theory Division, CERN, Geneva 23, Switzerland \\ STEFAN POKORSKI \\ Inst. of Theoretical Physics, Warsaw University, Hoża 69, 00-681 Warsaw, Poland
}

\begin{abstract}
After reviewing briefly the upper bounds on the mass of the lightest Higgs boson in the most general unconstrained minimal supersymmetric extension of the Standard Model, we discuss various arguments which reduce the parameter space of the model and give stronger predictions for $M_{h}$. First, the constraints from the presently available experimental data are summarized. Next, the role of of several additional theoretical assumptions is studied, after extrapolating the model to high energy scales. The most important ones are: perturbative validity up to the GUT scale and the electroweak symmetry breaking. A Higgs boson with $M_{h^{0}}<100$ $\mathrm{GeV}$ is predicted in several scenarios. Its absence in that mass range will have important implications for the parameter space of the model.
\end{abstract}

\section{Introduction}

Spontaneous breaking of the electroweak gauge symmetry $S U(2) \times U(1)$ is now confirmed experimentally with one per mille accuracy (see the Chapter by A. Blondel). However, the actual mechanism of this symmetry breaking still remains unknown and waits for experimental discovery. This is, by far, the most central question to particle physics and, in particular, to the experimental programs at LEP 2 and the LHC. It is very likely that the understanding of the mechanism of the electroweak symmetry breaking will not only provide us with the missing link in the Standard Model but, also, will be an important bridge to physics beyond it.

The minimal model for spontaneous electroweak gauge symmetry breaking is the Higgs mechanism, whose minimal version (the minimal Standard Model) requires one scalar $S U(2)$ doublet (Higgs doublet). The scalar potential at

\footnotetext{
${ }^{a}$ To appear in "Perspectives on Higgs Physics II" ed. G.L. Kane, World Scientific, Singapore.

${ }^{b}$ On leave of absence from the Institute of Theoretical Physics, Warsaw University, Hoża 69, 00-681 Warsaw, Poland.
} 
some scale $\Lambda$ is:

$$
V(\Lambda)=m^{2}(\Lambda)|H(\Lambda)|^{2}+\frac{1}{2} \lambda(\Lambda)|H(\Lambda)|^{4}
$$

with the dependence on $\Lambda$ controlled by the renormalization group evolution (RGE). The mass of the physical scalar (Higgs boson) is $M_{\phi^{0}}^{2}=\lambda\left(M_{Z}\right) v^{2}\left(M_{Z}\right)$ where $v\left(M_{Z}\right)=\sqrt{4 M_{W}^{2} / g_{2}^{2}\left(M_{Z}\right)} \approx 246 \mathrm{GeV}$. There exist the well known theoretical bounds on the Higgs boson mass (see 1 for an extensive discussion) which follow from certain constraints on the behaviour of the self-coupling $\lambda(\Lambda)$. One can distinguish two types of bounds. The most general upper bound on $M_{\phi^{0}}$ follows from the requirement that the Standard Model is a unitary and weakly interacting theory at the energy scale $\mathcal{O}\left(M_{Z}\right)$. We get then $M_{\phi^{0}} \lesssim \mathcal{O}(1 \mathrm{TeV})$. Stronger bounds are $\Lambda$-dependent and are known under the names of the triviality (upper) bound and the vacuum stability (lower) bound. They follow respectively from the requirements that the theory remains perturbative $\left(\lambda(\Lambda)<16 \pi^{2}\right)$ and the vacuum remains stable $(\lambda(\Lambda)>0)$ up to a certain scale $\Lambda$. Those bounds are particularly interesting in the presence of the heavy top quark, $m_{t}=(175 \pm 6) \mathrm{GeV}$. They are shown in Fig. 3 in ref. 1 and lead to the striking conclusions: We see that the discovery of a light Higgs boson $\left(M_{\phi^{0}} \lesssim 80 \mathrm{GeV}\right)$ or a heavy one $\left(M_{\phi^{0}} \gtrsim 500 \mathrm{GeV}\right)$ would be a direct information about the existence of new physics below the scale $\Lambda \sim \mathcal{O}(1 \mathrm{TeV})$ (or at least of a strongly interacting Higgs sector). On the other hand, if the SM in its perturbative regime is to be valid up to very large scales $\Lambda$, of the order of the GUT scale $\Lambda \approx 10^{16} \mathrm{GeV}$, one gets strong bounds $140 \mathrm{GeV} \lesssim M_{\phi^{0}} \lesssim 180 \mathrm{GeV}$. In this case we face the well known hierarchy problem in the SM: $M_{\phi^{0}}, v \ll \Lambda$ and it is difficult to understand how the scalar potential remains stable under radiative corrections of the full theory. One way or another, the bounds on $M_{\phi^{0}}$ in the SM are strongly suggestive that the mechanism of spontaneous electroweak gauge symmetry breaking is directly related to the existence of a new scale (not much above the electroweak scale) in fundamental interactions. The central question can then be phrased as this: discover and investigate the next scale in fundamental interactions. Is it the scale of new strong interactions (strongly interacting Higgs sector or techicolour interactions or compositness scale) or the scale of soft supersymmetry breaking? We would like to stress the basic difference between these two lines of approach. In the first one, the new scale is also a cutoff scale for the perturbative validity of the electroweak theory. Supersymmetry offers a solution to the hierarchy problem while maintaining the perturbative nature of the theory up to the GUT or even Planck scale. This is a welcome feature if such facts as the gauge coupling unification are not to be considered as purely accidental. 
Another difference is in the expectations for the Higgs boson mass: in the strong interaction scenarios it is naturally heavy, with its mass close to the new scale $\Lambda$. In supersymmetric extensions of the SM the lightest Higgs scalar $h^{0}$ generically remains light, $M_{h^{0}} \sim \mathcal{O}(100 \mathrm{GeV})$ and only logarithmically correlated with the scale of the soft supersymmetry breaking.

It is the purpose of this Chapter to summarize the predictions for the Higgs sector in supersymmetric extensions of the SM. The Higgs sector in the Minimal Supersymmetric Standard Model (MSSM), considered as a low energy effective theory with all free parameters totally unconstrained has been discused in detail in 2 . For the sake of easy reference and to establish our notation we summarize those results in Section 2. The main conclusion of that Section is the specification of the set of parameters which determine the Higgs boson masses and the existence of general upper bounds for the mass of the lightest supersymmetric Higgs boson $h^{0}$. Next, in the main part of this article, we collect the available results and arguments which constrain the general parameter space relevant for the Higgs mass in the MSSM and, therefore, give more specific predictions for $M_{h^{0}}$. One should stress that our interest in the MSSM is well motivated. It is structured in such a way that the success of the $\mathrm{SM}$ in describing the precision electroweak data is maintained. Moreover, its virtue is that it can be extrapolated up to the large energy scales (the scales where the soft supersymmetry breaking terms are generated) in unambigous and quantitative way. We shall mainly consider the supergravity scenario for supersymmetry breaking in which the MSSM is extrapolated up to the GUT scale. A brief discussion of the gauge mediated symmetry breaking models 3 is, however, also included. Broadly speaking, the weak scale - large scale connection is the main source of constraints on the Higgs sector parameter space which we are going to present. Finally, in Section 5, we discuss the predictions for the Higgs sector in non-minimal versions of the supersymmetric extensions of the Standard Model.

\section{Higgs sector in MSSM - a brief summary}

In the Minimal Supersymmetric Standard Model the Higgs sector is particularly simple and predictive. Supersymmetry and the minimal particle content imply that it consists of two Higgs doublets, each coupled to only one type $\left(H_{1}\left(H_{2}\right)\right.$ couples to the down (up)) of fermions. The scalar Higgs potential reads:

$$
\begin{aligned}
V= & m_{1}^{2} \bar{H}_{1} H_{1}+m_{2}^{2} \bar{H}_{2} H_{2}+m_{3}^{2}\left(\epsilon_{a b} H_{1}^{a} H_{2}^{b}+c . c\right) \\
& +\frac{1}{8}\left(g_{1}^{2}+g_{2}^{2}\right)\left(\bar{H}_{1} H_{1}-\bar{H}_{2} H_{2}\right)^{2}+\frac{1}{2} g_{2}^{2}\left|\bar{H}_{1} H_{2}\right|^{2}
\end{aligned}
$$


where $\epsilon_{12}=-1$ and $m_{1}^{2}, m_{2}^{2}$ and $m_{3}^{2}$ are the soft supersymmetry breaking mass parameters. The crucial point about the potential (2) is that its quartic couplings are the electroweak gauge couplings (i.e. there is no $F$-term contribution to the scalar Higgs potential). The only free parameters are the three mass parameters. The tree level mass eigenstates of the Higgs bosons are: two $C P$-even $\left(h^{0}, H^{0}\right)$, one $C P$-odd $\left(A^{0}\right)$ and 2 charged $\left(H^{ \pm}\right)$physical particles and three Goldstone bosons "eaten up" by the gauge bosons. An important parameter is $\tan \beta \equiv v_{2} / v_{1}$ where $v_{i}$ minimize the tree level potential (2) and are given by $v_{1}=v \cos \beta, v_{2}=v \sin \beta$ with

$$
\begin{gathered}
v^{2}=\frac{8}{g_{1}^{2}+g_{2}^{2}} \frac{m_{1}^{2}-m_{2}^{2} \tan ^{2} \beta}{\tan ^{2} \beta-1} \\
\sin 2 \beta=\frac{2 m_{3}^{2}}{m_{1}^{2}+m_{2}^{2}}
\end{gathered}
$$

Since $v$ is fixed by the $Z^{0}$ mass, all physical Higgs boson masses are expressed in terms of only two free parameters. They can be taken e.g. as $\tan \beta$ and the mass $M_{A^{0}}^{2}$ of the $C P$-odd Higgs scalar $A^{0}$ given by $M_{A^{0}}^{2}=m_{1}^{2}+m_{2}^{2}$. The $C P$-even Higgs boson masses then read:

$$
M_{h^{0}, H^{0}}^{2}=\frac{1}{2}\left(M_{A^{0}}^{2}+M_{Z^{0}}^{2}+\sqrt{\left(M_{A^{0}}^{2}-M_{Z^{0}}^{2}\right)^{2}-4 M_{A^{0}}^{2} M_{Z^{0}}^{2} \cos ^{2} 2 \beta}\right)
$$

leading to the bound $M_{h^{0}}<M_{Z^{0}}$ and to the "natural" (i.e. independent of any other parameters) relation $M_{h^{0}}^{2}+M_{H^{0}}^{2}=M_{A^{0}}^{2}+M_{Z^{0}}^{2}$. The other relation is $M_{H^{ \pm}}^{2}=M_{W^{ \pm}}^{2}+M_{A^{0}}^{2}$.

The origin and the magnitude of radiative corrections to the Higgs boson masses can be easily understood. Let $M$ be the scale of the soft supersymmetry breaking sfermion masses. Neglecting terms suppressed by inverse powers of $M$, the dominant one-loop corrections to the effective potential $V_{\text {eff }}$, due to the top and stop loops, can be absorbed into renormalization of the parameters in the Higgs potential. One gets:

$$
\begin{aligned}
& V=\tilde{m}_{1}^{2} \bar{H}_{1} H_{1}+\tilde{m}_{2}^{2} \bar{H}_{2} H_{2}+\tilde{m}_{3}^{2}\left(\epsilon_{a b} H_{1}^{a} H_{2}^{b}+c . c\right) \\
& +\lambda_{1}\left|H_{1}\right|^{4}+\lambda_{2}\left|H_{2}\right|^{4}+\lambda_{3}\left|H_{1}\right|^{2}\left|H_{2}\right|^{2}+\lambda_{4}\left|\bar{H}_{1} H_{2}\right|^{2}
\end{aligned}
$$

The appearence of other couplings is protected by the symmetries of the model. It is clear on the dimensional grounds that

$$
\delta m_{i}^{2}=\tilde{m}_{i}^{2}-m_{i}^{2} \sim \mathcal{O}\left(M^{2}\right)
$$


They are logarithmically divergent but can be absorbed into the free parameters of the model. The corrections $\delta \lambda_{i}$ defined by

$$
\begin{array}{r}
\delta \lambda_{1}=\lambda_{1}-\frac{1}{8}\left(g_{1}^{2}+g_{2}^{2}\right), \quad \delta \lambda_{2}=\lambda_{2}-\frac{1}{8}\left(g_{1}^{2}+g_{2}^{2}\right) \\
\delta \lambda_{3}=\lambda_{3}+\frac{1}{4}\left(g_{1}^{2}+g_{2}^{2}\right), \quad \delta \lambda_{4}=\lambda_{4}-\frac{1}{2} g_{2}^{2}
\end{array}
$$

are all $\mathcal{O}(\log M)$. Moreover, from the non-renormalization theorem, the corrections $\delta \lambda_{i}$ are calculable (finite) in terms of the remaining parameters of the model. From the top and stop loops with attached four Higgs boson legs one gets

$$
\delta \lambda_{i} \sim \frac{12}{16 \pi^{2}} h_{t}^{4} \log \left(\frac{M_{\tilde{t}}^{2}}{m_{t}^{2}}\right)
$$

where $h_{t}$ is the top quark Yukawa coupling (factor 12 comes from 4 top degrees of freedom multipied by the color factor of 3 ) and $M_{\tilde{t}}$ denotes the scale of the stop masses. Thus, the correction to the $h^{0}$ mass is

$$
\delta M_{h^{0}}^{2} \sim \mathcal{O}\left(\frac{6 g_{2}^{2}}{16 \pi^{2}} \frac{m_{t}^{4}}{M_{W}^{2}} \log \left(\frac{M_{\tilde{t}}^{2}}{m_{t}^{2}}\right)\right)
$$

In general, taking into account the full structure of the stop mass matrix, the lightest Higgs boson mass in the MSSM is parametrized by

$$
M_{h^{0}}=M_{h^{0}}\left(M_{A^{0}}, \tan \beta, m_{t}, M_{\tilde{t}_{1}}, M_{\tilde{t}_{2}}, A_{t}, \mu, \ldots\right)
$$

where $M_{\tilde{t}_{i}}$ are the physical stop masses, $A_{t}$ and $\mu$ determine their mixing angle (as well as some of their trilinear couplings to the Higgs bosons) and ellipsis stand for other parameters whose effects are not dominant (e.g. the sbottom sector parameters).

In Fig. 17a we show $M_{h^{0}}$ as a function of $M_{A^{0}}$ for two values of $\tan \beta$ and $M_{\tilde{t}_{1}}=M_{\tilde{t}_{2}}=1 \mathrm{TeV}, \mu=0$ and two values of the $A_{t}$ parameter. We see that maximal $M_{h^{0}}$ is always obtained for $M_{A^{0}} \gg M_{Z^{0}}$ (in practice, the bound is saturated for $M_{A^{0}} \gtrsim 250 \mathrm{GeV}$ ). In this limit one gets from the effective potential approach (see ref. 2 for details) particularly simple result for the one-loop corrected $M_{h^{0}}$ E:

$M_{h^{0}}^{2}=M_{Z^{0}}^{2} \cos ^{2} 2 \beta+\frac{3 \alpha}{4 \pi s_{W}^{2}} \frac{m_{t}^{4}}{M_{W}^{2}}\left[\log \left(\frac{M_{\tilde{t}_{1}}^{2} M_{\tilde{t}_{2}}^{2}}{m_{t}^{4}}\right)+\left(\frac{M_{\tilde{t}_{1}}^{2}-M_{\tilde{t}_{2}}^{2}}{2 m_{t}^{2}} \sin ^{2} 2 \theta_{\tilde{t}}\right)^{2}\right.$ 

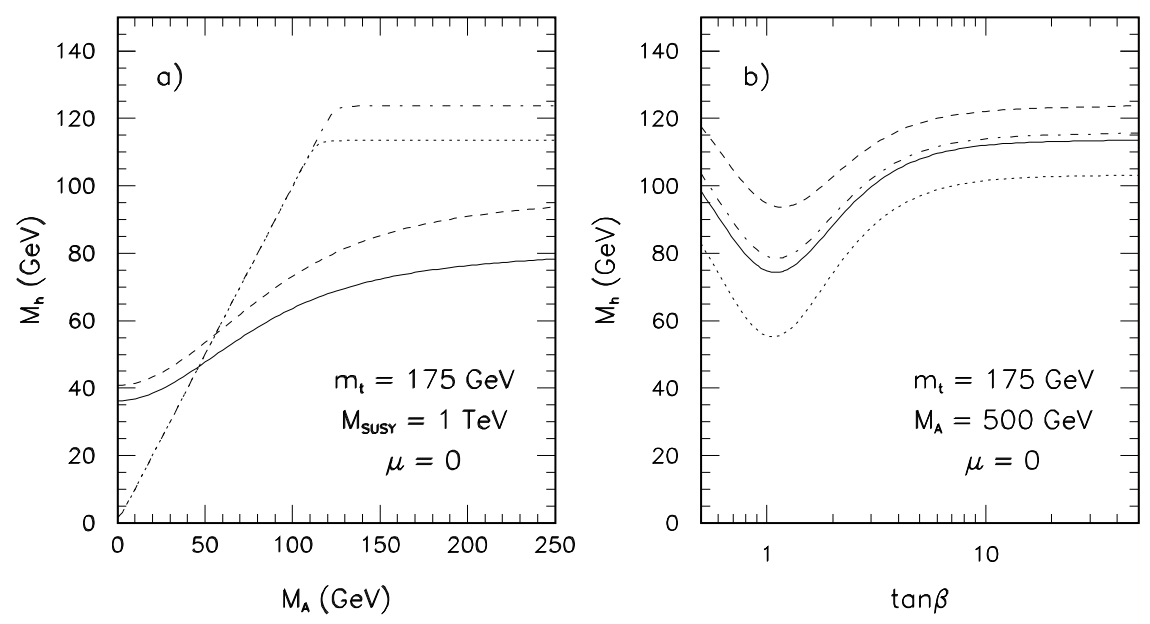

Figure 1: Radiatively corrected $M_{h}$ in the MSSM (1- and 2-loops). a) As a function of the $C P$-odd Higgs mass for $M_{S U S Y}=1 \mathrm{TeV}$ and for $\tan \beta=1.5$ (solid and dashed lines) and $\tan \beta=50$ (dotted and dash-dotted lines). Lower (upper) lines correspond to $A_{t}=0$ $\left(2.5 M_{S U S Y}\right)$ b) As a function of $\tan \beta$ for $m_{Q}=m_{U}=1 \mathrm{TeV}, A_{t}=0(2.5 \mathrm{TeV})$ solid (dashed) line and for $m_{Q}=500, m_{U}=100 \mathrm{GeV}, A_{t}=0(1 \mathrm{TeV})$ dotted (dash-dotted) line.

$$
\left.\times f\left(M_{\tilde{t}_{1}}^{2}, M_{\tilde{t}_{2}}^{2}\right)+\frac{M_{\tilde{t}_{1}}^{2}-M_{\tilde{t}_{2}}^{2}}{2 m_{t}^{2}} \sin ^{2} 2 \theta_{\tilde{t}} \log \left(\frac{M_{\tilde{t}_{1}}^{2}}{M_{\tilde{t}_{2}}^{2}}\right)\right]
$$

where $f(x, y)=2-(x+y) /(x-y) \log (x / y)$. For large $M_{A^{0}}$ the separate dependence on the parameters $A_{t}$ and $\mu$ has disappeared and is replaced by the effective dependence on the left-right stop mixing angle $\theta_{\tilde{t}}$. One should also mention (see2) that the two-loop corrections to $M_{h^{0}}$ are typically $\mathcal{O}(20 \%)$ of the one-loop corrections and are negative. In Figs. 1 and 2 they are taken into account in the approach proposed in 6 . The dependence on $\tan \beta$, illustrated in Fig. 1 b, is important for our further discussion.

\section{Experimental constraints on the parameters of the MSSM}

Having recalled the general parameter set relevant for the Higgs sector in supersymmetric models we proceed now to discuss constraints on those parameters which follow from various additional considerations. 

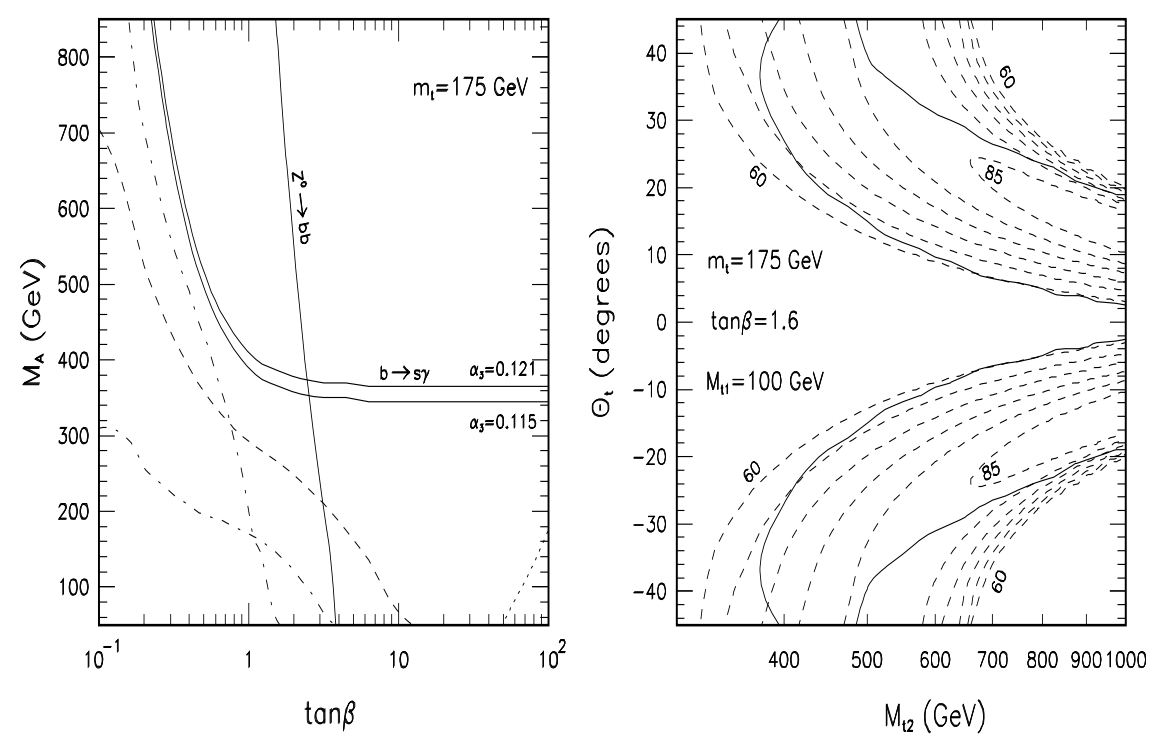

Figure 2: a) Bounds on the $C P$-odd Higgs boson mass in the MSSM with very heavy spectrum for $\alpha_{3}=0.115$ and 0.121 (solid lines) and (for $\alpha_{3}=0.117$ ) for the chargino and stop masses 500 and $250 \mathrm{GeV}$ - the dashed and dash-dotted lines respectively. The dotted line shows the constraint from $b \rightarrow c \tau \bar{\nu}_{\tau}$. Also shown are the constraints from $Z^{0} \rightarrow \bar{b} b$ in the MSSM with heavy spectrum (solid line) and for the chargino and stop masses equal 250 $\mathrm{GeV}$ (dashed line). b) Contours of the constant Higgs boson mass $M_{h^{0}}=60,65,70,75,80$ and 85 in the plane $\left(M_{\tilde{t}_{2}}, \theta_{\tilde{t}}\right)$ for $M_{A^{0}}=1000 \mathrm{GeV}, \tan \beta=1.6$ and the lighter stop mass equal $100 \mathrm{GeV}$. The region where $\Delta \chi^{2}<4$ (see the text) is bounded by the solid lines.

The parameters of the MSSM which enter into the prediction for $M_{h^{0}}$ are partially constrained by other experimental data. Although at present relatively weak, those are interesting constraints which correlate in the framework of the MSSM the Higgs boson mass(es) with other measurements without any additional assumptions. There are also stronger constraints which follow from embedding the MSSM into a SUSY GUT scenario, or more generally, from extrapolating the MSSM up to the GUT scale, supplemented with several plausible (simple) assumptions about physics at that scale. We begin our discussion with the former.

A number of experimental data constrains the tree level parameters $M_{A^{0}}$ and $\tan \beta$. These are, first of all, $B R(b \rightarrow s \gamma)$, $\Gamma\left(Z^{0} \rightarrow \bar{b} b\right)$ and (for large $\tan \beta) B R\left(b \rightarrow c \tau \bar{\nu}_{\tau}\right)$ id. Other processes (e.g. $B^{0}-\bar{B}^{0}$ mixing) give weaker 
constraints. The first two processes are sensitive to the $M_{H^{ \pm}}$and $\tan \beta$ via the charged Higgs boson - top quark loop contribution and the third one via the tree level $H^{ \pm}$exchange. The $b t H^{-}$coupling is given by

$$
\mathcal{L}_{b t H^{ \pm}}=\frac{g_{2}}{\sqrt{2} M_{W}} H^{+} \bar{\psi}_{t}\left(m_{t} \cot \beta P_{L}+m_{b} \tan \beta P_{R}\right) V_{t b} \psi_{b}+\text { h.c. }
$$

where $P_{L, R} \equiv\left(1 \mp \gamma^{5}\right) / 2$ (the coupling $b c H^{ \pm}$is similar with $\psi_{t} \rightarrow \psi_{c}$ and $\left.V_{t b} \rightarrow V_{c b}\right)$ and the coupling $\tau \nu_{\tau} H^{ \pm}$is given by:

$$
\mathcal{L}_{\tau \nu_{\tau} H^{ \pm}}=\frac{g_{2}}{\sqrt{2}} \frac{m_{\tau}}{M_{W}} \tan \beta H^{+} \bar{\psi}_{\nu_{\tau}} P_{R} \psi_{\tau}+\text { h.c. }
$$

We see that $B R\left(b \rightarrow c \tau \bar{\nu}_{\tau}\right)$ can be enhanced for large $\tan \beta$ and light $H^{ \pm}$and the measurement $B R\left(b \rightarrow c \tau \bar{\nu}_{\tau}\right)=2.69 \pm 0.44 \%$ gives the bound 10

$$
\tan \beta \lesssim 0.52 \frac{M_{H^{ \pm}}}{1 \mathrm{GeV}}
$$

which is essentially independent of the other parameters of the MSSM and is shown in Fig. 2a by the dotted line.

The first two processes get contributions also from diagrams with superpartners in the loop (the dominant one may come from the chargino-stop loop, due to the large Yukawa coupling for the higgsino component with the righthanded stop) and, in consequence, the exclusion regions in the $\left(M_{A^{0}}, \tan \beta\right)$ plane depend on supersymmetric parameters. The strongest dependence is on the $m_{C^{ \pm}}, M_{\tilde{t}_{i}}, \theta_{t}$. In the limit of very heavy superpartners $\left(m_{C_{i}^{ \pm}}, M_{\tilde{t}_{i}} \gtrsim 1\right.$ $\mathrm{TeV}$ ) we get the exclusion limits shown in Fig. 2a by the solid curves. The dependence of these limits on $m_{C_{1}^{ \pm}}, M_{\tilde{t}_{1}}$ (with all other superpartners at 1 $\mathrm{TeV}$ ) is illustrated by the dashed (dot-dashed) curve which is obtained for $m_{C_{1}^{ \pm}}=M_{\tilde{t}_{1}}=500(250) \mathrm{GeV}$. In each case a scan over the chargino composition and the left-right mixing angle in the stop sector have been performed.

Negative contribution of a light charged Higgs boson - top quark loop to $R_{b} \equiv \Gamma\left(Z^{0} \rightarrow \bar{b} b\right) / \Gamma\left(Z^{0} \rightarrow\right.$ hadrons $)$ becomes too large for small values of $\tan \beta$ (see eq. (13) $)$ excluding, in the MSSM with heavy spectrum, the region of the $\left(\tan \beta, M_{A^{0}}\right)$ plane to the left of the almost vertical solid line shown in Fig. 2a (we require that $R_{b}$ remains within $2 \sigma$ of the presently measured value $R_{b}^{\exp }=$ $0.2179 \pm 0.001211$ ). Positive contribution of the chargino - stop loop weakens significantly this bound (if both are light) as shown for $m_{C_{1}^{ \pm}}=M_{\tilde{t}_{1}}=250$ $\mathrm{GeV}$ by the almost vertical dot-dashed line. Similar effects are observed for $B R(b \rightarrow s \gamma)$. In the limit of heavy superpartners one obtains strong bound

${ }^{c}$ We require the branching ratio computed in the NLO approximation to fall into the interval $1 \times 10^{-4}<B R(b \rightarrow s \gamma)<4 \times 10^{-4}$ All uncertainties of the computation (for detailed discussion of the uncertainties see ref. 12 ) are taken into account. 
on $M_{A^{0}}$ : the $H^{ \pm}$- top loop adds positively to the SM contribution which, by itself, is in the upper edge of the experimentally allowed region. The bound is weakened in the presence of a light chargino - stop loop (which can interfere negatively with the $H^{ \pm}$-top loop) and even totally disappears for large values of $\tan \beta$. One should note, however, that for $\tan \beta \sim m_{t} / m_{b}$ the interference term is generically very large and consistency with the data requires a large amount of fine-tuning in the $\left(\theta_{\tilde{t}}, M_{\tilde{t}_{1}}\right)$ parameter space 13 . Thus, one concludes that the large $\tan \beta$ scenario is unlikely to be consistent with a light $C P$-odd Higgs boson. For $M_{h}$ this implies the plateau region in Fig. 17a. (The present experimental bound 4 is $M_{A^{0}} \gtrsim 55 \mathrm{GeV}$ for $\tan \beta \gtrsim 50$.)

Radiative corrections to $M_{h}$ are mainly dependent on the stop masses (for large $\tan \beta$ also on the sbottom mass) and on the parameters $A_{t}$ and $\mu$ (in the large $M_{A^{0}}$ limit they depend only on the combination $A_{t}+\mu \cot \beta$ which can be traded for $\theta_{\tilde{t}}$ as in eq. (12)) which are constrained by the precision data. A light left-handed stop would introduce additional source of the custodial $S U_{V}(2)$ breaking. Since the SM fit to the LEP precision data is very good with the $S U_{V}(2)$ breaking given mainly by the $t-b$ mass spliting, additional sources of the custodial $S U_{V}(2)$ breaking would tend to destroy the quality of the SM fit. In Fig. $2 \mathrm{~b}$ we show by the solid lines the limits in the $\left(M_{\tilde{t}_{2}}\right.$, $\theta_{\tilde{t}}$ ) plane for $M_{\tilde{t}_{1}}=100 \mathrm{GeV}$ obtained from the requirement that $\Delta \chi^{2}<4$ compared with the minimum of a fit to the electroweak observables found for heavy $\tilde{t}_{2}$. Similar bounds exist for heavier $\tilde{t}_{1}$. In Fig. 2b b, those limits are shown together with the contours of constant $M_{h^{0}}$.

In summary, the present experimental data do not significantly improve the general upper bound on $M_{h^{0}}$. However they give constraints on SUSY parameters and there are interesting correlations between parameter regions allowed by other processes (e.g. a light $C P$-odd Higgs boson is consistent with $B R(b \rightarrow s \gamma)$ and $\Gamma\left(Z^{0} \rightarrow \bar{b} b\right)$ only if chargino and stop are also light) and the Higgs boson mass $M_{h^{0}}$.

\section{The weak scale - large scale connection}

The parameter space of the low energy MSSM can be further reduced by introducing additional theoretical ideas. The first one involves the extrapolation of

\footnotetext{
${ }^{d}$ Those results are obtained under the assumption that the chargino -stop - bottom coupling is given by the Kobayashi-Maskawa angles. In principle, off-diagonal terms in the right-handed stop mass matrix are possible which would change those couplings. Such $(1,3)$ and $(2,3)$ terms are not constrained by any other data. However, other flavour off diagonal terms are strongly constrained so the presence of large $(1,3)$ and $(2,3)$ terms for right-handed squarks would mean strong flavour dependence in the squark mass matrices.
} 
the MSSM up to very high energy scales (the "desert" hypothesis) and the observation that to a very good approximation the $S U(3) \times S U(2) \times U(1)$ gauge couplings converge to a common value. With the supersymmetry breaking scale of order $1 \mathrm{TeV}$ or less the unification takes place at an energy scale of order $10^{16} \mathrm{GeV}$ and depends weakly on the details of the GUT-scale theory. One can rephrase this result by saying that e.g. the Weinberg angle is correctly predicted in terms of the measured values of $\alpha$ and $\alpha_{3}$ from the hypothesis of the gauge coupling unification. This is one of the most compelling hints for the low energy supersymmetry and, this should be strongly stressed, for the fact that physics remains perturbative up to the GUT scale $\sim 10^{16} \mathrm{GeV}$. Once assumed, the perturbative validity of the MSSM up to the scale $\sim 10^{16} \mathrm{GeV}$ has several interesting implications for the behaviour of the third generation Yukawa couplings and for the "interesting" (i.e. most plausible?) values of $\tan \beta$ and, in consequence, also for $M_{h^{0}}$.

The first important notion is that of the quasi-infrared fixed point for the top quark (or top and bottom quarks for large $\tan \beta$ ) Yukawa coupling. We recall the fixed point structure of the top quark Yukawa coupling $Y_{t} \quad\left(Y_{t} \equiv\right.$ $\left.h_{t}^{2} / 4 \pi\right)$ in the MSSM. The renormalization group equations have the form:

$$
\begin{aligned}
\frac{d Y_{t}}{d t} & =Y_{t}\left(a_{i}^{u} \alpha_{i}-c_{t} Y_{t}\right) \\
\frac{d \alpha_{i}}{d t} & =-b_{i} \alpha_{i}^{2}
\end{aligned}
$$

where $2 \pi t=\log \left(M_{G U T} / Q\right), \quad a_{i}^{u}=(13 / 15,3,16 / 3), b_{i}=(11,1,-3), c_{t}=6$ and $\alpha_{i}=g_{i}^{2} / 4 \pi$. Ignoring the smaller electroweak couplings, $Y_{t}$ is related to the QCD coupling $\alpha_{3}$ : the fixed point solution for the ratio $Y_{t} / \alpha_{3}$ reads 15 :

$$
Y_{t}^{F}(t)=\frac{a_{3}^{u}+b_{3}}{c_{t}} \alpha_{3}(t)
$$

One can also solve eqs. (16) explicitly 16 :

$$
Y_{t}(t)=\frac{4 \pi Y_{t}(0) E(t)}{4 \pi+c_{t} Y_{t}(0) F(t)}
$$

with

$$
E(t) \approx\left(\frac{\alpha_{3}(0)}{\alpha_{3}(t)}\right)^{\frac{a_{3}^{u}}{b_{3}}}, \quad F(t)=\int_{0}^{t} E\left(t^{\prime}\right) d t^{\prime}
$$

It may happen that $Y_{t}^{F}(t)$ is not reached because of too short a running but, nevertheless, $c_{t} Y_{t}(0) F(t) \gg 4 \pi$ and

$$
Y_{t}(t) \approx Y_{t}^{Q F}(t)=\frac{4 \pi E(t)}{c_{t} F(t)}
$$



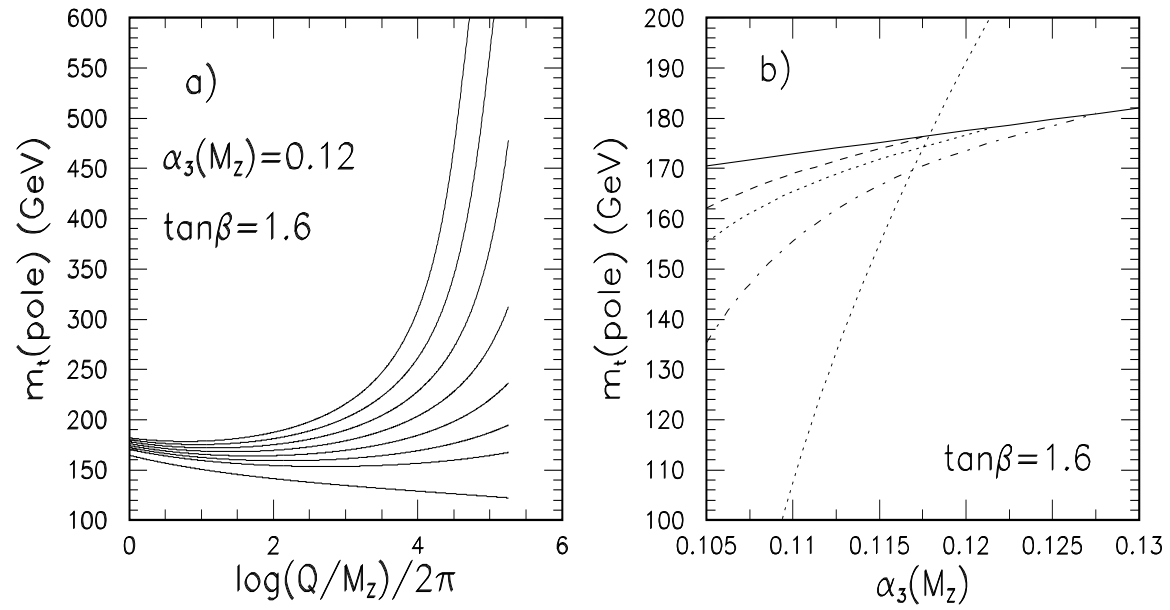

Figure 3: a) Top quark mass RG running for different boundary conditions at the scale $2 \times 10^{16} \mathrm{GeV}$. b) Q-IR top quark mass prediction (solid line) and top quark masses necessary to achieve bottom-tau Yukawa coupling unification for $m_{b}($ pole $)=4.7$ (dashed line), 4.9 (dotted) and 5.2 (dot-dashed) GeV. Gauge coupling unification favours the region to the right of the second dotted line.

i.e.the low energy value of $Y_{t}$ no longer depends on the initial value $Y_{t}(0)$. This is called the quasi-infrared fixed point solution (Q-IR) 17 and we have:

$$
Y_{t}^{Q F}(t) \approx Y_{t}^{F}(t) \frac{1}{1-\left(\frac{\alpha_{3}(t)}{\alpha_{3}(0)}\right)^{1+\frac{a_{3}^{u}}{b_{3}}}}
$$

This situation indeed occurs in the MSSM (for $b_{3}, a_{3}^{u}, c_{t}$ of the MSSM) for $Y_{t}(0) \gtrsim \mathcal{O}(0.1)$ (see Fig. 3a) i.e. for the initial values still in the perturbative regime! Thus, not only $Y_{t}^{Q F}\left(M_{Z}\right)$ is the upper bound for $Y_{t}\left(M_{Z}\right)$ but it can be reached at the limit of perturbative physics 18 . The Q-IR prediction for the running top quark mass in the $\bar{M} S$ scheme, for $\alpha_{3}\left(M_{Z}\right) \bar{n}^{0.11-0.13}$ and small and moderate values of $\tan \beta$ is approximately given by 19 :

$$
m_{t}^{Q F}\left(m_{t}\right) \approx 196 \mathrm{GeV}\left[1+2\left(\alpha_{3}\left(M_{Z}\right)-0.12\right)\right] \sin \beta
$$

The physical top quark mass (pole mass) is obtained by including QCD corrections which contribute $\mathcal{O}(10 \mathrm{GeV})$ to the final result. Eq. 222 combined 
with $m_{t}=(175 \pm 6) \mathrm{GeV}$ gives us a lower bound on $\tan \beta: \tan \beta \gtrsim 1.4$ Similar results are also found in the large $\tan \beta$ region in which both the top and bottom Yukawa couplins are large]. In the numerical calculation two-loop RG equations are used.

It is clear from eq. (22) that the experimental value of $m_{t} \approx 175 \mathrm{GeV}$ is very close to its perturbative upper bound in the MSSM. To know how close, we need to know $\tan \beta$ but one is tempted to speculate that $m_{t} \approx m_{t}^{Q F}$ and then $\tan \beta$ must be either close to its lower bound or very large.

An interesting observation is that the GUT assumption about unification of the bottom and tau Yukana couplings gives independent support to the idea that $Y_{t}\left(M_{Z}\right)=Y_{t}^{Q F}\left(M_{Z}\right)^{23,24}$. Quantitatively, this conclusion depends on the values of $\alpha_{3}\left(M_{Z}\right)$ and $m_{b}$ (pole) and on the threshold corrections to the relation $Y_{b}=Y_{\tau}$. Generically, however, strong interaction renormalization effects for $Y_{b}$ are too strong and large top quark Yukawa coupling contribution to the running of $Y_{b}$ is needed to balance them (see Fig. 3 3 b). For $Y_{b}=Y_{\tau}$ within $10 \%, \alpha_{3}\left(M_{Z}\right)>0.11$ and $m_{b}($ pole $)<5.2 \mathrm{GeV}$ one gets $m_{t} \approx m_{t}^{Q F}$ within $10 \%$. Thus, the possibility of $m_{t} \approx m_{t}^{Q F}$ is supported by several independent arguments (also models for dynamical determination of $Y_{t}$ give values close to the IR fixed point 25 ) and, together with the measured value of $m_{t}=175 \pm 6$ $\mathrm{GeV}$, it makes the region $1.5 \lesssim \tan \beta \lesssim 2$ particularly interesting.

Another interesting region of $\tan \beta$ is $\tan \beta \approx m_{t} / m_{b}$. Large values of $\tan \beta$ have been discussed for some time as a solution to the $m_{t} / m_{b}$ hierarchy with full unification of the third generation Yukawa couplings $Y_{t}=Y_{b}=Y_{\tau}$ 26.27. Sich unification is predicted e.g. by simple versions of the SUSY $S O(10)$ GUT2628 and remains to be an interesting possibility.

In conclusion, although one cannot rule out intermediate values of $\tan \beta$, the arguments given above are compelling enough to consider the low and large $\tan \beta$ regions as "interesting" ones. In those regions the Higgs boson masses are bounded more strongly than in the general MSSM. For $m_{t}=175$ $\mathrm{GeV}$, from Fig. $\mathrm{fb}$ we see that if $Y_{t}$ is $\lesssim 10 \%$ away from its IR value (which corresponds to $\tan \beta \lesssim 1.85$ ) we have $M_{h^{0}} \lesssim 100 \mathrm{GeV}$. For large $\tan \beta$ we have $M_{h^{0}} \approx M_{A^{0}}$ up to $\sim 120 \mathrm{GeV}$ and for heavier $M_{A^{0}}$ (for arguments in favour of such $M_{A^{0}}$, see the previous Section) the $M_{h^{0}}$ remains constant (independent of $M_{A^{0}}$ ) with the value fixed by radiative corrections.

${ }^{e}$ This bound can be slightly lowered to $\tan \beta \gtrsim 1.1$, in gauge mediated SUSY breaking models, due to the presence of additional coloured matter fields at the intermediate scale $M \sim 10^{5}-10^{7} \mathrm{GeV}$.

${ }^{f}$ In this case the bottom pole mass may due to the supersymmetric loop correction 2d, 21. Similar corrections may be even important for the Kobayashi-Maskawa mixing angles 22

${ }^{g}$ For large $\tan \beta$ this conclusion may be less strong. 
Finally, we are going to discuss several constraints on the range of the soft supersymmetry breaking masses $m_{1}^{2}, m_{2}^{2}, m_{3}^{2}$, the top squark masses and the mixing parameters $A_{t}, \mu$ (i.e. the remaining parameters relevant for $M_{h}$ ) which follow from the extrapolation of the MSSM to high energies. They are particularly interesting and easy to discuss under the assumption that the $h_{t}$ is not too far from its quasi-infrared fixed point limit. For the sake of definitness we shall mainly focus on the low and intermediate $\tan \beta$ region and present analytic results in the 1-loop approximation24,29. More complete 2-loop numerical calculations 24 . 19 confirm very well these analytic considerations.

In the 1-loop approximation and expanding in $y(t) \equiv Y_{t}(t) / Y_{t}^{Q F}(t)$ the RG equations for the dimensionful parameters can be solved analytically 2429 . Denoting by $\bar{m}^{2}(t) \equiv m_{Q}^{2}(t)+m_{U}^{2}(t)+m_{H_{2}}^{2}(t), 2 \pi t \equiv \log \frac{M}{Q}$ with $M=M_{G U T}$ or any intermediate scale, and with $m_{K}^{2}, K=H_{i}, Q, U, D, L, E$, standing for the soft supersymmetry breaking mass parameters of the Higgs, left-handed squark, right-handed up-type squark, right-handed down-type squark, lefthanded slepton and right-handed slepton, respectively we get the the following results:

$$
\begin{aligned}
\bar{m}^{2}(t) & =(1-y) \bar{m}^{2}(0)-y(1-y) A_{t}(0)\left(A_{t}(0)-2 \hat{\xi} M_{1 / 2}\right) \\
& +\left(\bar{\eta}-y \hat{\eta}+y^{2} \hat{\xi}^{2}\right) M_{1 / 2}^{2} \\
m_{K}^{2}(t)= & m_{K}^{2}(0)-\frac{c_{K}}{c_{t}} y \bar{m}^{2}(0)-\frac{c_{K}}{c_{t}} y(1-y) A_{t}(0)\left(A_{t}(0)-2 \hat{\xi} M_{1 / 2}\right) \\
+ & {\left[\eta_{K}-\frac{c_{K}}{c_{t}}\left(y \hat{\eta}+y^{2} \hat{\xi}^{2}\right)\right] M_{1 / 2}^{2}+D_{K}, } \\
& D_{K}=-\kappa_{K} \bar{m}_{Y}^{2}(0)\left[1-\left(\frac{\alpha_{1}(0)}{\alpha_{1}(t)}\right)^{-13 / 33}\right] \\
\bar{m}_{Y}^{2}(t) & \equiv-m_{H_{1}}^{2}(t)+m_{H_{2}}^{2}(t) \\
& +\sum_{g e n}\left[m_{E}^{2}(t)-m_{L}^{2}(t)+m_{Q}^{2}(t)+m_{D}^{2}(t)-2 m_{U}^{2}(t)\right] .
\end{aligned}
$$

Functions $\eta_{K}(t), \hat{\xi}(t), \hat{\eta}(t)\left(\bar{\eta} \equiv \eta_{Q}+\eta_{U}+\eta_{H_{2}}\right)$ are given in closed forms in terms of integrals over the gauge couplings and are defined in the Appendix of ref. 29. For $M=2 \times 10^{16} \mathrm{GeV}$ and $\alpha_{3}\left(M_{Z}\right)=0.12$ they take values: $\hat{\xi}=2.23, \hat{\eta}=12.8, \eta_{Q}=7.04, \eta_{U}=6.62, \eta_{H_{1}}=\eta_{H_{2}}=0.513$ The coefficients 
$c_{K}$ and $\kappa_{K}$ read: $c_{Q}=1, c_{U}=2, c_{H_{2}}=3, c_{L}=c_{E}=c_{D}=c_{H_{1}}=0$; $\kappa_{H_{1}}=-\kappa_{H_{2}}=\kappa_{L}=-3 / 26, \kappa_{E}=3 / 13, \kappa_{Q}=1 / 26, \kappa_{U}=-2 / 13, \kappa_{D}=1 / 13$. The evolution of the trilinear couplings $A_{k}$ are given by:

$$
A_{i}(t)=A_{i}(0)-\frac{C_{i}}{c_{t}} y A_{t}(0)+\left(\frac{C_{i}}{c_{t}} y \hat{\xi}-\xi_{i}\right) M_{1 / 2},
$$

Here $C_{t}=c_{t}=6, C_{b}=1$ and $C_{\tau}=0$. Factors $\xi_{i}(t)$ are defined in the Appendix of ref. 29 and for $M=2 \times 10^{16} \mathrm{GeV}$ and $\alpha_{3}\left(M_{Z}\right)=0.12 \xi_{t}=3.97$. Quantities at $t=0$ are the initial values of the parameters at the scale $M$, $M_{1 / 2} \equiv M_{3}(0)$ is the initial gaugino (gluino) mass (computing numerical values of $\hat{\xi}, \hat{\eta} \ldots$ we have assumed that $\left.M_{1} / \alpha_{1}(0)=M_{2} / \alpha_{2}(0)=M_{3} / \alpha_{3}(0)\right)$.

There are several interesting observations about solutions (23-27). Firstly, to a very good approximation squark mass parameters of the first two generations decouple from the running of the masses $m_{H_{1}}^{2}$ and $m_{H_{2}}^{2}$ (they enter only through small hypecharge $D$-term (25)). As stressed in refs. 30,29 , this is very important for the "naturalness" problem. Moreover, we observe interesting "fixed point" behaviour for the parameter $A_{t}$ which, in the limit $y \rightarrow 1$, becomesindependent of its initial values and fixed in terms of the gaugino mass $M_{1 / 2} 24$ (unless $A_{t}(0) \gg M_{1 / 2}$ but large values of $A_{t}(0)$ are constrained by the requirement of the absence of the colour breaking minima).

Having relations 23-27) we can discuss the impact of the requirement of the proper electroweak symmetry breaking on the low energy parameter space, under various assumptions about the pattern of the soft supersymmetry breaking parameters at large scale. This requirement correlates the low energy soft supersymmetry breaking masses in the Higgs potential with the values of $M_{Z^{0}}$ and $\tan \beta$, as given in eqs. (3, 1, and, in turn, with other parameters which enter into their RGE. Actually, under each specific assumption about the scale of supersymmetry breaking and the pattern of the soft terms, one can perform a global analysis which includes the electroweak breaking and the existing experimental constraints, and obtain the predictions for the Higgs sector. We present here the results of such an analysis for the following three scenarios: two supergravity scenarios with the GUT relation for the gaugino masses $M_{1}=$ $M_{2}=M_{3} \equiv M_{1 / 2}$ : one with universal soft scalar masses (and universal $A$ terms) and one with universal scalar masses in $S O(10)$ multiplets i.e. with universal sfermion masses (and universal $A$-terms) and two soft Higgs boson masses as independent parameters, and the gauge mediated supersymmetry breaking scenario. Part of the most important experimental constraints has already be discussed in Section 3. In the global analysis discussed now, we also include the following bounds: $m_{C^{ \pm}}>85 \mathrm{GeV}, \Gamma\left(Z^{0} \rightarrow N_{1}^{0} N_{1}^{0}\right)<4 \mathrm{MeV}$, $B R\left(Z^{0} \rightarrow N_{1}^{0} N_{2}^{0}\right)<10^{-4}$. 
There has been often addressed the question of fine-tuning (large cancellations)inthe Higgs potential in models with the soft terms generated at large scales $31,30,32$. Indeed, if supersymmetry is to be the solution to the hierarchy problem in the SM, it should not introduce another fine-tuning in the Higgs potential. The origin of the problem is easy to see. Combining (3) and 23 27) we can express $M_{Z^{0}}$ for a given $\tan \beta$ in terms of the initial values $m_{K}^{2}(0)$, $M_{1 / 2}$ and the $\mu$ parameter:

$$
\begin{aligned}
M_{Z^{0}}^{2} & =-2 \mu^{2}(t)+a_{H_{1}} m_{H_{1}}^{2}(0)+a_{H_{2}} m_{H_{2}}^{2}(0)+a_{Q U}\left(m_{Q}^{2}(0)+m_{U}^{2}(0)\right) \\
& +a_{A A} A_{t}^{2}(0)+a_{A M} A_{t}(0) M_{1 / 2}+a_{M} M_{1 / 2}^{2}
\end{aligned}
$$

For $m_{t}=175 \mathrm{GeV}$ the generic values of the coefficients in eq. (28) in the supergravity scenario e.g. for $\tan \beta \approx 1.65(2.2)$ corresponding to $y \approx 0.95(0.85)$ are $a_{H_{1}} \approx 1.2(0.5), a_{H_{2}} \approx 1.7(1.5), a_{Q U} \approx 1.5(1.1), a_{A A} \approx 0.1(0.2), a_{A M} \approx$ $-0.3(-0.7), a_{M} \approx 15.0(10.8)$. Eq. 28$)$ shows that for values of $\mu, M_{1 / 2}$ and $/$ or $m_{K}^{2}(0)$ much larger than $M_{Z^{0}}$ one needs large cancellations. Asymptotically, we are back to the hierarchy problem in the SM. Although the idea of "naturalness" is only qualitative, one can at least correlate the magnitude of the necessary cancellations with the values of the parameters $\mu, m_{K}^{2}(0)$ and $M_{1 / 2}$ and, in consequence, with the low energy mass parameters. One notes, in particular, that the smalness of $a_{Q U}$ (compared to $a_{M}$ ) puts weaker constraints on the "natural" values of $m_{K}^{2}(0)$ than large $a_{M}$ does on $M_{1 / 2}$. However, in the physical spectrum this effect is partially counterbalanced by the fact that the stop soft masses tend to be suppressed compared to $m_{Q, U}(0)$ by the running with large top quark Yukawa coupling. This effect is stronger for the right handed stop than for the left handed one and gives the hierarchy $M_{\tilde{t}_{R}}<M_{\tilde{t}_{L}}$. Important source of fine-tuning can also be the relation (4) which correlates the values of $\tan \beta$ and the $B_{0}$ parameter.

"Naturalness" of a given parameter set can be quantified e.g. bycalculating the derivatives of $M_{Z^{0}}^{2}$ with respect to the soft mass parameters 31 30.

$$
\Delta_{i} \equiv\left|\frac{a_{i}}{M_{Z}^{2}} \frac{\partial M_{Z}^{2}}{\partial a_{i}}\right|
$$

(other criteria have also been proposed 32 but will not be discussed here since, at any rate, the concept of naturalness is only qualitative.)

In the global analysis, which combines the electroweak breaking with experimental constraints, it is interesting to check the "naturalness" of different parameter regions i.e. to check the values $\left(\Delta_{i}\right)$ for each parameter set. Before presenting the results for the three scenarios considered, it is worthwile to remember several general remarks. First, as already said, the "naturalness" 
criterion is only a qualitative one and it is unclear how big cancellations are "acceptable" ones. The hierarchy problem in the SM means fine-tuning of many orders of magnitude and from that perspective cancellations of one, two or even three orders of magnitude are still very small. Secondly, we do not know the theory in which soft supersymmetry breaking terms originate and it may well be that such a theory will give them correlated to each other, thus "explaining" the cancellations between them. Finally one can see from eqs. (3,14) and (28) that the necessary cancellations tend to increase in the small and large $\tan \beta$ regions advocated as the most interesting ones on the basis of earlier arguments. Indeed, the coefficients $a_{i}$ in eq. (28) have $1 /\left(\tan ^{2} \beta-1\right)$ singularity. Moreover, both for small and very large $\tan \beta$ eq. (44) gives very strong dependence on $B_{0}$ (i.e. large derivative with respect to $B_{0}$ in eq. (29)).

With those comments in mind we now present the results of our global analysis, for the three scenarios considered and for several values of $\tan \beta$. In each case the lightest Higgs boson mass is shown as a function of the heavier stop and the maximal and minimal values of $\left(\Delta_{i}\right)^{\max }$ for the points on each plot are also noted.

Widely discussed has been the so-called minimal supergravity model (Ansatz) with universal scalar and gaugino masses and universal trilinear soft terms. In this model all superpartner masses are given in terms of five parameters: $m_{0}^{2}, M_{1 / 2}, \mu, A_{0}$ and $B_{0}$. Two of them can be traded for $M_{Z^{0}}$ and $\tan \beta$. Thus, we get strongly correlated superpartner spectrum and correlated with the Higgs boson masses. It is now particularly simple to follow our global analysis and to determine the allowed range of the lightest Higgs boson mass as a function of the heavier stop mass. In Fig. 1 we show the results for $\tan \beta=1.65$ and 2.5 (corresponding to $y \approx 0.95(0.80)$ ). The parameter space has been scanned up to $M_{\tilde{t}_{2}}=1 \mathrm{TeV}$ with $\mu_{0}$ and $B_{0}$ fixed by $M_{Z^{0}}$ and $\tan \beta$. We see that, in this model, requiring the proper breaking of the electroweak symmetry and with the imposed experimental constraints the lightest Higgs boson mass is bounded from below: $M_{h^{0}} \gtrsim 75(85) \mathrm{GeV}$ for $\tan \beta=1.65(2.5)$ (for $\tan \beta=10$ the lower bound is around $105 \mathrm{GeV}$ ).

The model also gives lower bound on $M_{A^{0}}$ of about $500 \mathrm{GeV}$ at $\tan \beta=$ 1.65 and decreasing to $300 \mathrm{GeV}$ at $\tan \beta=10$. The heavier stop is bounded from below at $\sim 450 \mathrm{GeV}$. Of course, the crucial role in obtaining those bounds is played by the universality Ansatz combined with the existing experimental constraints. We note also an interesting dependence on the sign of the $\mu$ parameter (two clear branches in Fig. Ta) which is a reflection of the acceptable region in the $\left(\theta_{\tilde{t}}, M_{\tilde{t}_{2}}\right)$ plane shown in Fig. $2 \mathrm{~b}$. The mass $M_{h^{0}}$ is bounded from above at 95,105 and $120 \mathrm{GeV}$ for $\tan \beta=1.65,2.5$ and 10 , respectively. Thus the general bounds shown in Fig 1 b can be reached even in this constrained 

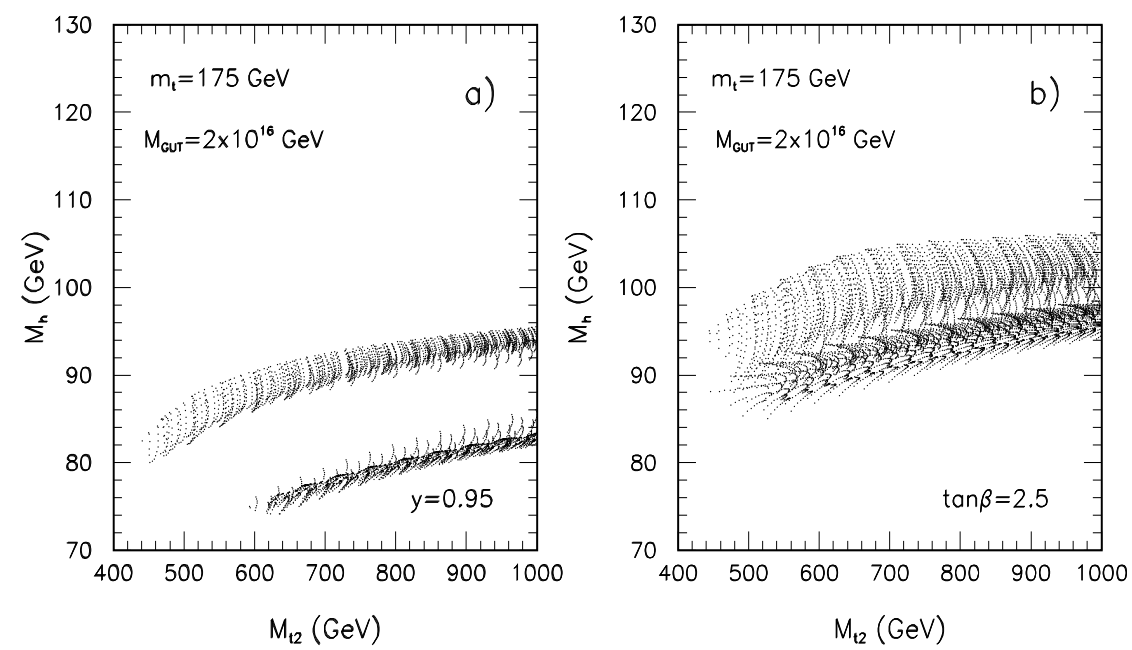

Figure 4: $M_{h^{0}}$ versus the mass of the heavier stop $\left(M_{\tilde{t}_{2}}\right)$ obtained from universal boundary conditions at the scale $2 \times 10^{16} \mathrm{GeV}$ a) close to the Q-IR fix point of the top quark mass $(y \approx 0.95) \quad$ b) for $\tan \beta=2.5(y \approx 0.8)$. In both cases no fine-tunig criterion is imposed.

model.

Turning now to the fine-tuning problem we observe first that the model does not admit at all solutions with all $\Delta_{i}<1$. This is mainly because of the imposed experimental limit $m_{C^{ \pm}}>85 \mathrm{GeV} 30$ which pushes $M_{1 / 2}$ into the region with $\Delta_{M_{1 / 2}} \gtrsim 10$ for all $\tan \beta$ values ?. Moreover, close to the IR fixed point (for $\tan \beta \approx 1.65$ ), there do not even exist solutions with all $\Delta_{i}<100$ 24. Actually, defining two different $\Delta$ 's: $\Delta_{\max }^{\prime} \equiv \max \left\{\Delta_{M_{1 / 2}}, \Delta_{m_{0}}, \Delta_{\mu_{0}}\right\}$ and $\Delta_{\max } \equiv \max \left\{\Delta_{\max }^{\prime}, \Delta_{B_{0}}, \Delta_{A_{0}}\right\}$, the points in Fig. Ga(b) give $70(28) \lesssim$ $\Delta_{\text {max }}^{\prime} \lesssim 970(560), \quad 130(35) \lesssim \Delta_{\max } \lesssim 5400(750)$. As expected from the general arguments, cancellations become weaker with increasing $\tan \beta$. In Fig. 5 a (b) we show the results for $\tan \beta=2.5(10)$ with the cut $\Delta_{\max }<100$. We note that in this case such a cut leaves a non-empty parameter region but gives stronger upper bounds on the Higgs boson mass for the same values

\footnotetext{
${ }^{h}$ Strictly speaking, this conclusion is valid as potential renormalized at $M_{Z^{0}}$. It is well known 33 34, 32 30 that inclusion of the full 1-loop corrections to the scalar potential diminishes somewhat the degree of fine-tuning and the bound $m_{C^{ \pm}}>85 \mathrm{GeV}$ becomes marginally consistent with $\Delta_{i}<10$, for intermediate values of $\tan \beta$. We neglect this effect here, as it does not change our qualitative conclusions.
} 

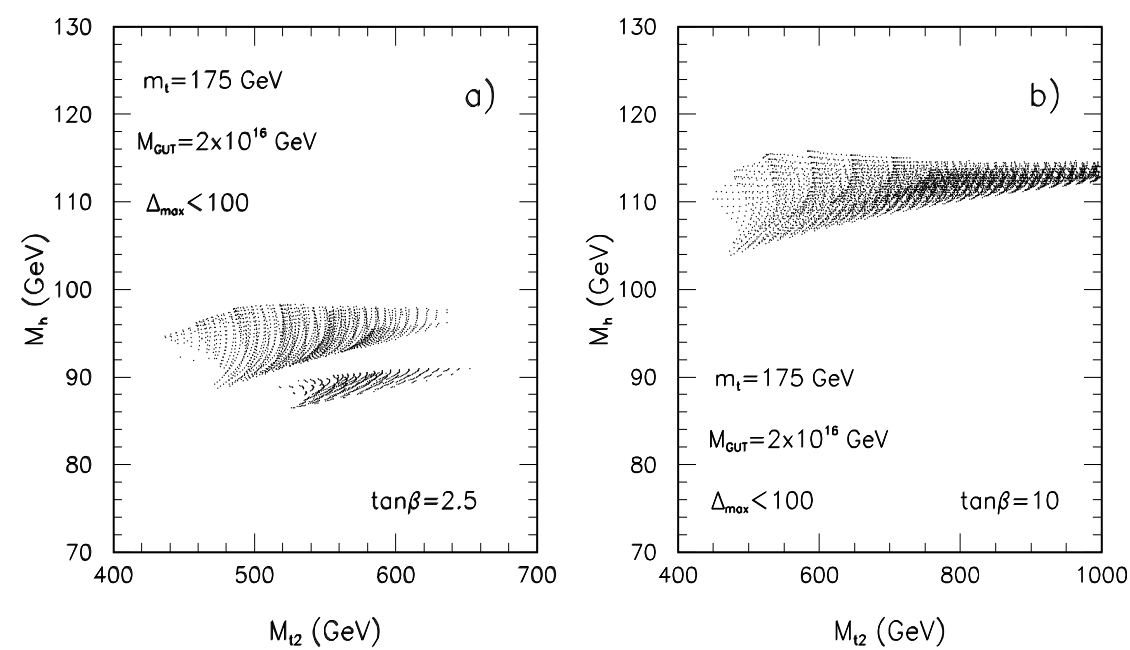

Figure 5: $M_{h^{0}}$ versus the mass of the heavier stop $\left(M_{\tilde{t}_{2}}\right)$ obtained from universal boundary conditions at the scale $2 \times 10^{16} \mathrm{GeV}$ and requiring $\Delta_{i}<100$ a) for $\tan \beta=2.5(y \approx 0.80)$ b) for $\tan \beta=10(y \approx 0.71)$.

of $M_{\tilde{t}_{2}}$. They result mainly from the bound on $A_{0}$ (i.e. on the left-right mixing) obtained due to increasing $\Delta_{M_{1 / 2}}$ with increasing $A_{0}$. Moreover, the cut $\Delta_{\max }<100$ gives also an upper bound on $M_{\tilde{t}_{2}}$. A weaker cut, $\Delta_{\max }^{\prime}<$ 100 , does not change the results for $\tan \beta=10$ (as expected) but allows for broader range of $M_{\tilde{t}_{2}}$ for $\tan \beta=2.5$ (with the upper bound for $M_{h^{0}}$ as for $\left.\Delta_{\max }<100\right)$. Finally we note one more interesting effect: a cut on $\Delta$ 's gives almost flat (instead of logarithmic) dependence of $M_{h^{0}}$ on $M_{\tilde{t}_{2}}$. An increase in $M_{\tilde{t}_{2}}$ is balanced by a decrease in $A_{0}$ (i.e. in the left-right mixing) to keep $\Delta$ 's below the imposed bound.

In the next step one can study a less restrictive model, with the pattern of the soft terms consistent with the $S O(10)$ unification, i.e. with the universal sfermion masses and the two Higgs boson masses taken as independent parameters. It turns out that the predictions the $M_{h^{0}}, M_{A^{0}}$ and $M_{\tilde{t}_{2}}$ (as well as the degree of fine-tuning) are very similar to those in the universal case and need not be independently shown here. This similarity can be partly understood in terms of the important role played by the limit $m_{C^{ \pm}}>85 \mathrm{GeV}$ and by the constraints from $b \rightarrow s \gamma$ and from precision data, which are not sensitive to the assumed non-universality in the Higgs boson mass parameters. Moreover, 
there is no real change in the values of $\Delta_{i}$ 's since their expected decrease with the increasing number of free parameters is now reduced by the dissapearance of certain cancellations in $\Delta_{i}$ 's which are present in the universal case. Finally, the considered values of $\tan \beta$ are enough above 1 to make the electroweak symmetry breaking easy even in the universal case and the considered breakdown of full universality does not significantly enlarge the parameter space consistent with the electroweak symmetry breaking. Thus, the results presented in Figs. 4 and 5 are representative also for the considered partial breakdown of universality.

The difference between universal and non-uniersal boundary conditions becomes more visible in the large $\tan \beta$ region 35.20 . In the latter case the parameters $\Delta$ are typically $\sim \tan \beta$ whereas in the former $-\mathcal{O}(1000)$, i.e. finetuning of order $10^{-3}$. From the point of view of the predictions for the MSSM spectrum, the large $\tan \beta$ region is characterized by the expectation of a relatively light $C P$-odd Higgs boson, $M_{A^{0}} \lesssim \mathcal{O}(200 \mathrm{GeV})$. For further details in the large $\tan \beta$ region, in particular for correlations with radiative corrections to the $b$-quark mass and with $b \rightarrow s \gamma$ decay, we refer the reader to 35.20 .21 .

Finally, it is interesting to compare the supergravity scenario with models in which supersymmetry breaking is transmitted to the observable sector through ordinary $S U(3) \times S U(2) \times U(1)$ gauge interactions of the so-called messenger fields at scales $M \ll M_{G U T}$. In general, these gauge-mediated models of SUSY breaking are characterized by two scales: the scale $M$, which is of the order of the average messenger mass and the scale $\sqrt{F}(\sqrt{F}<M)$ of supersymmetry breaking. Messenger fields are assumed to form complete $\mathbf{5}+\overline{\mathbf{5}}$ $($ or $\mathbf{1 0}+\overline{\mathbf{1 0}}) S U(5)$ representations. Their number $n$ is restricted to $n_{\max }=4$ by the requirement of perturbativity of the gauge couplings up to the GUT scale. In those models the LSP is a very light gravitino $\left(m_{\tilde{G}}<1 \mathrm{keV}\right)$. For $\sqrt{F}<10^{6} \mathrm{GeV}$ the decay length of the lightest neutralino into a photon and gravitino is such that this decay occurs within a typical detector. Hence, photons + missing energy become a signature of supersymmetry at LEP and Tevatron colliders. The absence of such events in the existing data strongly disfavours charginos and stops with masses below $\sim 125 \mathrm{GeV}$ and $\sim 140 \mathrm{GeV}$ respectively 36 .

In terms of $M$ and $x \equiv F / M^{2}$ the soft supersymmetry breaking parameters of the MSSM at the scale $\sim M$ are given by:

$$
\begin{gathered}
M_{i}=\frac{\alpha_{i}(M)}{4 \pi} M n x g(x) \equiv \frac{\alpha_{i}(M)}{4 \pi} M y \\
m_{\tilde{f}}^{2}=2 M^{2} n x^{2} f(x) \sum_{i}\left(\frac{\alpha_{i}(M)}{4 \pi}\right) C_{i}=2 M^{2} y^{2} z \sum_{i}\left(\frac{\alpha_{i}(M)}{4 \pi}\right) C_{i}
\end{gathered}
$$


where $C_{3}=4 / 3, C_{2}=3 / 4, C_{1}=(3 / 5) Y^{2}$ ( $Y$ being the hypercharge of the scalar $\tilde{f})$, the functions $g(x)$ and $f(x)(g(0)=f(0)=1, g(1) \approx 1.4, f(1) \approx$ $0.70)$ can be found in ref. 37 and the factor $z \equiv f(x) / n g^{2}(x)$. Thus, for fixed messenger sector (i.e. fixed $n$ ) and fixed scale $M$ all soft supersymmetry breaking masses are predicted in terms of $y\left(0<y<n_{\max } g(1) \approx 5.6\right) \|$. In those models we also have $A_{0} \approx 0$ as the $A_{0}$ parameter can be generated at two loop only 38 . However, the values of the soft masses $m_{H_{1,2}}^{2}$ may differ significantly from their values given by eq. (31) since they can be modified by physics involved in generation of $B_{0}$ and $\mu_{0}$ parameters 39 . Therefore, in our scans we take $y, m_{H_{1}}, m_{H_{2}}, \mu_{0}$ and $B_{0}$ as free parameters (the last two are fixed by $M_{Z^{0}}^{2}$ and $\tan \beta$ ). To be general, the factor $z$ in eq. (31) is scanned between $z_{\min }=f(1) / n_{\max } g^{2}(1) \approx 0.15$ and $z_{\max }=1$. For definitness we will consider $M=10^{5} \mathrm{GeV}$ only.

Here we follow the same simple approach we used for the supergravity models. On the parameter space consistent with the electroweak symmetry breaking we impose the discussed earlier experimental constraints (now we require $m_{C^{ \pm}}>120 \mathrm{GeV}, M_{\tilde{t}_{1}}>140 \mathrm{GeV}$ ). Very important rôle is played by $b \rightarrow s \gamma$. The requirement of good $b \rightarrow s \gamma$ rate reduces otherwise rather widely spread out $h^{0}$ and $A^{0}$ Higgs boson masses (for $\tan \beta=2.5$ : $20<M_{h^{0}}<100$ $\mathrm{GeV})$ to a narrow band $\left(80<M_{h^{0}}<100 \mathrm{GeV}, M_{A^{0}}>200 \mathrm{GeV}\right)$. This effect can be easily understood (see Fig. 2) because in the model considered squarks and charginos are rather heavy ] so a light $A^{0}$ is not allowed by $b \rightarrow s \gamma$ and light $h^{0}$ is always associated with light $A^{0}$. Moreover, surviving small values of $M_{h^{0}}(\sim 80 \mathrm{GeV}$ for $\tan \beta=2.5$ are associated with lowest values of $M_{\tilde{t}_{2}}(\lesssim 500 \mathrm{GeV})$ which are eliminated by imposing the $\Delta \chi^{2}<4$ cut. Finally, if we also require "naturalness" e.g. by demanding $\Delta_{\max }<100$ $\left(\Delta_{\max }=\max \left\{\Delta_{x}, \Delta_{m_{H 1}}, \Delta_{m_{H 2}}, \Delta_{\mu_{0}}, \Delta_{B_{0}}\right\}\right)$, we constrain the heavier stop mass $M_{\tilde{t}_{2}}$ and $C P$-odd higgs boson mass $M_{A^{0}}$ from above to $\lesssim 700 \mathrm{GeV}$.

Final results are shown in Fig. 6 as a plot of $M_{h^{0}}$ versus the mass of the heavier stop $M_{\tilde{t}_{2}}$ predicted in models of gauge mediated supersymmetry breaking with $M=10^{5} \mathrm{GeV}$ for $\tan \beta=2.5$ and 10 . As in the case of supergravity models, the restriction of the chargino and stop masses eliminates solutions with $\Delta_{\max }<10$. With all constraints imposed, $M_{h^{0}}$ turns out to be surpris-

\footnotetext{
${ }^{i}$ Nonzero lower bound on $y$ is set by Tevatron limit on gluino mass, $m_{\tilde{g}} \gtrsim 150 \mathrm{GeV}$, which is, however, subject to some restrictions

${ }^{j}$ In addition, because $\mu$ values required by electroweak symmetry breaking are large, the lighter chargino turns out to have only small higgsino component and hence its $b \tilde{t} C^{-}$ coupling is weaker than that of the pure higgsino chargino which is responsible for the limit shown in Fig. 2

${ }^{k}$ turalness of the gauge mediated models has been analyzed by different methods in refs. 40 .
} 

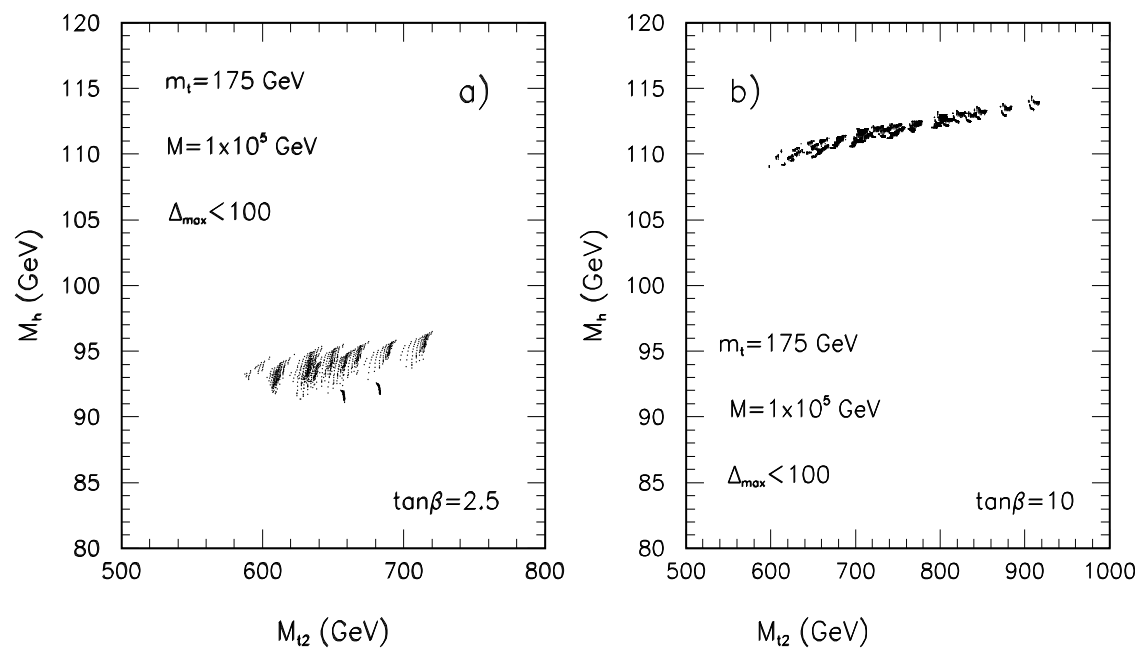

Figure 6: Results for gauge mediated supersymmetry breaking models with $M=10^{5} \mathrm{GeV}$ for $\tan \beta=2.5$ : a) $M_{h^{0}}$ versus the mass of the heavier stop $\left(M_{\tilde{t}_{2}}\right)$ b) versus the mass of the $C P$-odd Higgs boson $\left(M_{A^{0}}\right)$. Condition $\Delta_{i}<100$ is imposed.

ingly tightly constrained. For $\tan \beta=2.5(10)$ values of the lightest scalar Higgs boson are bounded by $90(108) \mathrm{GeV} \lesssim M_{h^{0}} \lesssim 97(115) \mathrm{GeV}$. Masses of the $C P$ odd Higgs boson are bounded to $280(200) \mathrm{GeV} \lesssim M_{A^{0}} \lesssim 700(850) \mathrm{GeV}$. These upper bounds should be compared to the ones obtained in 11 in the restricted model of gauge mediated supersymmetry breaking (with $x=1, n=1$ and with $m_{H_{1,2}}$ as given by eq. (31)) without imposing any additional constraints. It is interesting that in the much more general scenario described above, after imposing experimental and naturalness cuts, one gets upper bounds on $M_{h^{0}}$ not higher than those obtained in 41 .

\section{$5 \quad$ Non-minimal SUSY models}

Non-minimal supersymmetric extensions of the Standard Model can go beyond the MSSM in several directions. Models with additional Higgs_singlets 4243 , doublets and/or triplets have been considered in the literature 4 . The gauge group of the MSSM can also be extended by e.g. additional $U(1)$ factors. Finally, models with $R$-parity spontaneously broken (Supersymmetric Singlet Majoron Model) have been proposed 45 . The important question is what can 
be said about the Higgs sector in such extensions.

In the most popular extension, the so called Next-to-Minimal Supersymmetric Standard Model (NMSSM) 42,43 , one introduces a gauge singlet superfield $N$ and replaces the term $\mu H_{1} H_{2}$ in the MSSM superpotential by

$$
\lambda H_{1} H_{2} N+\frac{\kappa}{3} N^{3}
$$

with the obvious motivation to avoid the $\mu$ problem 4 . The model has been analysed in several papers. At the tree level, the upper bound for the mass of the lightest Higgs boson

$$
M_{h^{0}}^{2} \leq M_{Z}^{2}\left(\cos ^{2} 2 \beta+\frac{2 \lambda^{2}}{g^{2}} c_{W}^{2} \sin ^{2} 2 \beta\right)
$$

has been derived 4344. It is important that this bound depends neither on the sfermion masses (which can be much larger than $M_{Z}$ ) nor on the vacuum expectation value of the singlet field $N$ (which is not constrained by $M_{Z}$ ). Therefore, the bound is controlled only by the dimensionless Yukawa coupling $\lambda$ which is constrained by the requirement of the perturbativity of the model, $\lambda \lesssim 4 \pi$.

This result, that the tree level bound does not depend on the parameters which can be of order of the supersymmetry breaking scale (and, hence, is always $\lesssim \mathcal{O}\left(G_{F}^{-1 / 2}\right)$ ), has been extended to madels containing arbitrary numbers of Higgs singlets, doublets and triplets 44 47. Finally if was also shown to be valid in the Supersymmetric Single Majoron Model to . Almost modelindependent proofs of this remarkable fact has been given in 49.48 . Radiative corrections to the tree level bound 5 are under control as in the MSSM and has heen shown to depend on soft SUSY breaking masses only logarithmically 51. 52.48 .

The tree level bounds can be further strenghtened by requiring that the additional Yukawa couplings remain perturbative up to a scale $\Lambda$ (GUT scale). For the NMSSM this has been done in the papers 44.53.52. Analysing coupled RGE for $\lambda, Y_{t}$ and $\alpha_{s}$ the upper limit on $\lambda$ has been found as a function of $Y_{t}$ for $\Lambda=10^{16} \mathrm{GeV}$. For large values of $m_{t}$, for which $Y_{t}$ is always close to its perturbativity limit, the coupling $\lambda$ is forced to be small and hence its effects become less important. Including all relevant one-loop radiative corrections it was found that, for $m_{t}$ in the present experimental range, the upper bound for the lightest Higgs boson in the NMSSM is only $5-15 \mathrm{GeV}$ larger than the corresponding upper bound in the MSSM and, for $M_{\tilde{t}_{i}} \leq 1 \mathrm{TeV}$ it is smaller than $150 \mathrm{GeV}$. Effects of the additional assumptions like gauge coupling unification, universality of the soft SUSY breaking masses and requirement of 
the correct electroweak symmetry breaking have also been studied in this model 54.

\section{Summary}

In unconstrained minimal supersymmetric extension of the Standard Model there exists the well known upper bound on the mass of the lightest supersymmetric Higgs. The available parameter space of the model is now considerably reduced by the existing experimental data and can be further reduced by additional theoretical assumptions, mostly related to the extrapolation of the model to very large energy scales. Especially fruitful is the assumption about perturbative validity of the model up to the GUT scale and the requirement of the proper electroweak breaking combined with a simple Ansatz (such as universality or partial universality) for the pattern of the soft supersymmetry breaking terms. Such a reduction in the parameter space results in more definite expectations for $M_{h^{0}}$ than the general bounds. Several arguments point toward $M_{h^{0}}<100 \mathrm{GeV}$. Both, the discovery or the absence of the Higgs boson in this mass range will have strong implications for the supersymmetric extension of the Standard Model.

\section{Acknowledgments}

Work supported by the Polish Commitee for Scientific Research under the grant 2 P03B 04012 and by the European Union under contract CIPD-CT940016. P.H.Ch. would like to thank Dr. M. Misiak for cross checking his code for $b \rightarrow s \gamma$.

\section{References}

1. M. Quiros this volume. The figure we refer to can be also found (fig.1) in Higgs Physics at LEP 2 M. Carena, P. Zerwas (convenors), CERN Yellow Report, G. Altarelli, F. Zwirner eds. (hep-ph/9602250).

2. H.E. Haber this volume.

3. M. Dine, W. Fischler, M. Srednicki Nucl. Phys. B189 (1981) 575; S. Dimopoulos, S. Raby Nucl. Phys. B192 (1981) 353; L. Alvarez-Gaumé, M. Claudson, M. Wise Nucl. Phys. B207 (1982) 96; M. Dine, A. Nelson Phys. Rev. D48 (1993) 1277; M. Dine, A. Nelson, Y. Shirman Phys. Rev. D51 (1995) 1362; M. Dine, A. Nelson, Y. Nir, Y. Shirman Phys. Rev. D53 (1996) 2658. 
4. S.P. Li, M. Sher Phys. Lett. B140 (1984) 339; H.-P. Nilles, M. Nusbaumer Phys. Lett. B145 (1984) 73; J. Gunion, H.E. Haber Nucl. Phys. B272 (1985) 1.

5. Y. Okada, M. Yamaguchi, T. Yanagida Prog. Theor. Phys. 85 (1991) 1; H.E. Haber, R. Hempfling Phys. Rev. Lett. 66 (1991) 1815; J. Ellis, G. Ridolfi, F. Zwirner Phys. Lett. B257 (1991) 83, 262 (1991) 477.

6. R. Hempfling Ph.D. Thesis, UCSC preprint SCIPP 92/28 (1992); J.L. Lopez, D.V. Nanopoulos Phys. Lett. 266B (1991) 397.

7. M. Carena, J.-R. Espinosa, M. Quiros, C.E.M. Wagner Phys. Lett. B355 (1995) 209; M. Carena, M. Quiros, C.E.M. Wagner Nucl. Phys. B461 (1996) 407.

8. R. Barbieri, G.-F. Giudice Phys. Lett. 309 (1993) 86; A.J. Buras, M. Misiak, M. Münz, S. Pokorski Nucl. Phys. B424 (1994) 374;

9. J. Rosiek Phys. Lett. 252B (1990) 135; A. Denner et al. Z. Phys. C51 (1991) 695; M. Boulware, D. Finnell Phys. Rev. D44 (1991) 2054.

10. P. Krawczyk, S. Pokorski Phys. Rev. Lett. 60 (1988) 182; G. Isidori Phys. Lett. 298B (1993) 409; Y. Grossman, H.E. Haber, Y. Nir Phys. Lett. 357B (1995) 630.

11. The Lep Electroweak Working Group, CERN preprint LEPEWWG/9602 .

12. K.G. Chetyrkin, M. Misiak, M. Münz preprint ZU-TH 24/96 (hep$\mathrm{ph} / 9612313)$.

13. P.H. Chankowski S. Pokorski Nucl. Phys. B475 (1996) 3.

14. The ALEPH collaboration, contribution to Int. Europhysics. Conf. on High Energy Physics, Brussels, Belgium July-August 1995, paper EPS0415.

15. B. Pendleton, G.G. Ross Phys. Lett. B98 (1981) 291; see also W. Zimmermann Comm. Math. Phys. 97 (1985) 211; J. Kubo, K. Sibold, W. Zimmermann Phys. Lett. B200 (1989) 191.

16. L. Ibañez, C. Lopez Nucl. Phys. B233 (1984) 511; L. Ibañez, C. Lopez, C. Muñoz Nucl. Phys. B256 (1985) 218; A. Bouquet, J. Kaplan, C.A. Savoy Nucl. Phys. B262 (1985) 299.

17. C.T. Hill Phys. Rev. D24 (1981) 691; C.T. Hill C.N. Leung, S. Rao Nucl. Phys. B262 (1985) 517.

18. L. Alvarez-Gaumé, J. Polchinski, M.B. Wise Nucl. Phys. B221 (1983) 495; J. Bagger, S. Dimopoulos, E. Masso Phys. Rev. Lett. 55 (1985) 920; M. Olechowski, S. Pokorski Phys. Lett. B257 (1991) 388; C.D. Frogatt, I.D. Knowles, R.G. Moorhouse Phys. Lett. B249 (1990) 273; M. Carena et al. Nucl. Phys. B369 (1992) 33.

19. M. Carena C.E.M. Wagner Nucl. Phys. B452 (1995) 45. 
20. L.J. Hall, R. Ratazzi, U. Sarid Phys. Rev. D50 (1994) 7048; R. Hempfling Phys. Rev. D49 (1994) 6168.

21. M. Carena, M. Olechowski, S. Pokorski, C.E.M. Wagner Nucl. Phys. B426 (1994) 269;

22. T. Blazek, S. Pokorski, S. Raby Phys. Rev. D52 (1995) 4151.

23. H. Arason et al. Phys. Rev. Lett. 67 (1991) 2933; S. Kelley, J.L. Lopez, D.V. Nanopoulos Phys. Lett. B278 (1992) 140; V. Barger, M.S. Berger, P. Ohmann Phys. Rev. D47 (1993) 1093; V. Barger, M.S. Berger, P. Ohmann, R.J.N. Phillips Phys. Lett. B314 (1993) 35; P. Langacker, N. Polonsky Phys. Rev. D47 (1993) 4028, D49 (1994) 1454; M. Carena, S. Pokorski, C.E.M. Wagner Nucl. Phys. B406 (1993) 59; W. Bardeen, M. Carena, S. Pokorski, C.E.M. Wagner Phys. Lett. B320 (1994) 110;

24. M. Carena, M. Olechowski, S. Pokorski, C.E.M. Wagner Nucl. Phys. B419 (1994) 213;

25. C. Kounnas, F. Zwirner, I. Pavel Phys. Lett. B335 (1994) 403; P. Binetruy, E. Dudas Phys. Lett. B338 (1994) 23.

26. T. Banks Nucl. Phys. B303 (1988) 172; M. Olechowski, S. Pokorski Phys. Lett. B214 (1988) 393; G.-F. Giudice, G. Ridolfi Z. Phys. C41 (1988) 447; B. Ananhtarayan, G. Lazarides, Q. Shafi Phys. Rev. D44 (1991) 1613; S. Dimopoulos, L.J. Hall, S. Raby Phys. Rev. Lett. 68 (1992) 1984, Phys. Rev. D45 (1992) 4192.

27. S. Pokorski in Proc. XII Int. Workshop on Weak Interactions and Neutrinos, Ginosar, Israel Nucl. Phys. Proc. Suppl. B13 (1990) 606; H.-P. Nilles in Proc. of the 1990 Theoretical Advanced Study Institute in Elementary Particle Physics, eds. M. Cvetic, P. Langacker, World Scientific, Singapore, p. 633; W. Majerotto, B. Mösslacher Z. Phys. C48 (1990) 273; M. Drees, M.M. Nojiri Nucl. Phys. B369 (1992) 54; B. Ananhtarayan, G. Lazarides, Q. Shafi Phys. Lett. B300 (1993) 245.

28. G.W. Anderson, S. Raby, S. Dimopoulos, L.J. Hall Phys. Rev. D47 (1993) 3702; G.W. Anderson et al. Phys. Rev. D49 (1994) 3660; L.J. Hall, S. Raby Phys. Rev. D51 (1995) 6524.

29. M. Carena et al. preprint CERN-TH/96-241 (hep-ph/9612261) Nucl. Phys. B in press.

30. S. Dimopoulos, G.-F. Giudice Phys. Lett. B357 (1995) 573.

31. R. Barbieri, G.-F. Giudice Nucl. Phys. B306 (1988) 63.

32. G.W. Anderson, D.J. Castaño Phys. Lett. B347 (1995) 300, Phys. Rev. D52 (1995) 1693; A. Strumia preprint FT-UAM-96-40 (hepph/9609286); G.W. Anderson, D.J. Castaño, A. Riotto Phys. Rev. D55 (1997) 2950.

33. M. Olechowski, S. Pokorski Nucl. Phys. B404 (1993) 590. 
34. B. de Carlos, J.A. Casas Phys. Lett. B309 (1993) 320.

35. M. Olechowski, S. Pokorski Phys. Lett. B344 (1995) 201; F. Borzumati, M. Olechowski, S. Pokorski Phys. Lett. B349 (1995) 311.

36. S. Ambrosanio et al. Phys. Rev. D54 (1996) 5395.

37. S. Dimopoulos, G.-F. Giudice, A. Pomarol Phys. Lett. B389 (1996) 37; S. Martin Phys. Rev. D55 (1997) 3177.

38. S. Dimopoulos, S. Thomas, J. Wells preprint SLAC-PUB-7237 (hep$\mathrm{ph} / 9609434)$.

39. G. Dvali, G.-F. Giudice, A. Pomarol Nucl. Phys. B478 (1996) 31.

40. P. Ciafaloni, A. Strumia preprint FT-UAM 96/43 (hep-ph/9611204); G. Bhattacharyya, A. Romanino preprint IFUP-TH 67/96 (hep$\mathrm{ph} / 9611243)$

41. A. Riotto, O. Törnqvist, R.N. Mohapatra Phys. Lett. B388 (1996) 599.

42. P. Fayet Nucl. Phys. B90 (1975) 104; H.-P. Nilles, M. Srednicki, D. Wyler Phys. Lett. B120 (1983) 346; J.-P. Derendinger, C.A. Savoy Nucl. Phys. B237 (1984) 307.

43. M. Drees Int. J. Mod. Phys. A4 (1989) 3635; J. Ellis, J. Gunion, H. Haber, L. Roszkowski, F. Zwirner Phys. Rev. D39 (1989) 844.

44. J.-R. Espinosa, M. Quiros Phys. Lett. B279 (1992) 92.

45. G.-F. Giudice, A. Masiero, M. Pietroni, A. Riotto Nucl. Phys. B396 (1993) 243.

46. L. Hall, J. Lykken, S. Weinberg Phys. Rev. D27 (1983) 2359; J.E. Kim, H.-P. Nilles Phys. Lett. B138 (1984) 150; K. Inoue, A. Kakuto, T. Takano Prog. Theor. Phys. 75 (1986) 664; A.A. Anselm, A.A. Johansen Phys. Lett. B200 (1988) 331; G.-F. Giudice, A. Masiero Phys. Lett. B206 (1988) 480.

47. J.-R. Espinosa, M. Quiros Phys. Lett. B302 (1993) 51.

48. J.-R. Espinosa Phys. Lett. B353 (1995) 243.

49. G.L. Kane, C. Kolda, J. Wells Phys. Rev. Lett. 70 (1993) 2686.

50. U. Ellwanger, M. Rausch de Traubenberg Z. Phys. C53 (1992) 521; U. Ellwanger, M. Lindner Phys. Lett. B301 (1993) 365; U. Ellwanger B303 (1993) 271; W.T.A. ter Veldhuis preprint PURD-TH-92-11 (hep$\mathrm{ph} / 9211281)$.

51. T. Elliott, S.F. King, P.L. White Phys. Lett. B305 (1993) 71, B314 (1993) 56, B351 (1995) 213.

52. T. Elliott, S.F. King, P.L. White Phys. Rev. D49 (1994) 2435.

53. P. Binetruy, C.A. Savoy Phys. Lett. B277 (1992) 453.

54. U. Ellwanger, M. Rausch de Traubenberg, C.A. Savoy Phys. Lett. B315 (1993) 331, Z. Phys. C67 (1995) 665; S.F. King, P.L. White Phys. Rev. D52 (1995) 4183. 